\title{
Cholinergic synaptic vesicles are metabolically and biophysically heterogeneous even in resting terminals
}

\author{
V.P. Whittaker \\ Arbeitsgruppe Neurochemie, Max-Planck-Institut für Biophysikalische Chemie, Göttingen (F.R.G.)
}

(Accepted 2 August 1989)

Key words: Synaptic vesicle; Metabolic heterogeneity; Cholinergic electromotor nerve terminal; Torpedo marmorata

\begin{abstract}
The metabolic heterogeneity of synaptic vesicles in the cholinergic nerve terminals of the electromotor neurons of Torpedo marmorata has been studied in resting tissue by evaluating the molecular acetylcholine content (MAC) of synaptic vesicles after extraction from frozen and crushed tissue and high-resolution centrifugal density gradient separation in a zonal rotor. Although vesicular acetylcholine was distributed in the gradient as a single, more or less symmetrical peak, 3 subpopulations of synaptic vesicles could be identified: a small, relatively light subpopulation of low MAC on the ascending limb of the acetylcholine peak, designated $\mathrm{V}_{0}$, a main population of fully charged vesicles designated $\mathrm{V}_{1}$, and a small, denser subpopulation also of low MAC on the descending limb of the acetylcholine peak, designated $\mathrm{V}_{2}$. The mean proportions and MACs of the 3 pools were: $V_{0}, 13 \%, 58,000 ; V_{1}, 53 \%, 246,000 ; V_{2}, 34 \%, 79,000$. When tritiated acetate was perfused through excised blocks of electric organ for 1-2 h before vesicle isolation, the specific radioactivity of the acetylcholine in the $V_{0}$ and $V_{2}$ pools was 10-30 times higher than in the $V_{1}$ pool. This suggests that both the $V_{0}$ and $V_{2}$ pools are not generated by the isolation procedure but are present in the intact endings and are functionally active. On the basis of their density and uptake of newly synthesized acetylcholine, the $V_{0}$ and $V_{2}$ pools were identified with the previously described $\mathrm{VP}_{0}$ pool of axonal vesicles and the $\mathrm{VP}_{2}$ pool of recycling vesicles in stimulated nerve terminals respectively. Since stimulation of electromotor nerve terminals is known to generate large proportions of $\mathrm{VP}_{2}$ vesicles, variations in the proportion of $\mathrm{V}_{2}$ vesicles in unstimulated tissue are attributed to varying amounts of adventitious stimulation of the tissue during dissection and perfusion.
\end{abstract}

\section{INTRODUCTION}

Historically, a difficulty for the synaptic vesicle theory of evoked transmitter release ${ }^{36}$ was the observation that, in synapses in which the transmitter pools had been labelled with radioactive precursors, the specific activity of released transmitter was usually considerably higher than that of the main fraction of isolated vesicles'. This paradox was resolved when it was found that synaptic vesicles are metabolically heterogeneous and that those vesicles actively taking up and releasing newly synthesized highly radioactive transmitter constituted a recycling subpopulation which could be separated from fully-charged, metabolically inert reserve vesicles by density-gradient centrifuging ${ }^{4,6,8,41}$ or particle exclusion chromatography ${ }^{17}$. This has been shown for mammalian brain $^{8}$ and myenteric plexus ${ }^{5}$ and Torpedo electromotor nerve terminals ${ }^{6,41}$. The two vesicle pools have been designated $\mathrm{VP}_{1}$ (reserve) and $\mathrm{VP}_{2}$ (recycling).

Although stimulation markedly increases the proportion of recycling vesicles in the total population of synaptic vesicles, recycling vesicles are also present in resting' tissue $e^{40,41}$. Thus, when synaptic vesicles are isolated from unstimulated blocks of Torpedo electric tissue perfused with radioactive choline or acetate and centrifugally separated on a density gradient, the single peak of vesicular acetylcholine often shows a more or less pronounced shoulder on its dense side and, although there has been relatively little uptake of radioactive transmitter, that in the shoulder region has a much higher specific radioactivity than the main peak. Since this is the region of the gradient from which recycling vesicles are recovered in preparations made from stimulated tissue, it seems reasonable to conclude that resting tissue too contains a pool of recycling vesicles. This may arise as a result of spontaneous release, as evinced by the occurrence of miniature postsynaptic potentials ${ }^{11,12,30}$ or adventitious stimulation during the removal of the tissue.

Evidence has recently been obtained, by pulse-labelling of vesicular proteoglycan, for the presence, in resting electromotor nerve terminals, of a pool of recently transported 'empty' synaptic vesicles (designated $\mathrm{VP}_{0}$ ) that are less dense than the main, transmitter-rich population, but equal in density to vesicles isolated from the electromotor axons ${ }^{21}$. The existence of such vesicles, and of $\mathrm{VP}_{2}$-type vesicles, in resting electromotor terminals is also suggested by the tendency of stable synaptic vesicle markers such as vesicular proteoglycan ${ }^{32}$ and the

Correspndence: V.P. Whittaker, Arbeitsgruppe Neurochemie, Max-Planck-Institut für Biophysikalische Chemie, Göttingen, F.R.G. 
putative vesicular ATP transporter ${ }^{23,31,32}$ to form a broader peak than acetylcholine in the density gradient.

The purpose of the present work was to analyse more fully the various pools of synaptic vesicles present in the resting cholinergic synapse, by making further use of this now well-established and highly reproducible technique of centrifugal density gradient separation in a zonal rotor, of cytoplasmic extracts of electric tissue, well known as a rich source of the purely cholinergic nerve terminals of electromotor neurons ${ }^{6,7,10,25,27,34,38,42}$. The distribution of synaptic vesicles in the gradients was compared with that of acetylcholine and the various vesicle pools were identified by their mean molecular acetylcholine contents.

\section{MATERIALS AND METHODS}

\section{Isolation and fractionation of synaptic vesicles}

The general procedure used here has already been fully described ${ }^{6}$. Briefly, live specimens of Torpedo marmorata obtained from the Station Biologique d'Arcachon were kept in tanks of circulated, aerated and filtered artificial sea water at $15-17^{\circ} \mathrm{C}$ until used. Electric organs were removed from fish under Tricaine anaesthesia induced by immersion in sea water containing $0.05 \%$ Tricaine methanesulphonate (Sandoz MS 222 supplied by Sigma, Deisenhofen, FRG) for $15-20 \mathrm{~min}$ by which time responses to tail-pinching had ceased. Suitably sized blocks of tissue (mean weight $60 \pm 7(4) \mathrm{g}$; here and elsewhere means are given \pm S.E.M. with the number of observations in parentheses) were frozen, either immediately or after perfusion at $20^{\circ} \mathrm{C}$ in a closed circuit for $1-2 \mathrm{~h}$ with Torpedo Ringer's solution (composition in $\mathrm{mM}: \mathrm{NaCl} 280, \mathrm{KCl}$ $3.5, \mathrm{MgCl}_{2} 1.2, \mathrm{CaCl}_{2} 3.4$, glucose 10 , urea 300 , sucrose 100 , $\mathrm{NaHCO}_{3} 5.0, \mathrm{NaH}_{2} \mathrm{PO}_{4} 1.2$, adjusted to $\mathrm{pH}$ 7.3) to which $\left[{ }^{3} \mathrm{H}\right.$ ]acetate $\left(2 \mu \mathrm{Ci} \cdot \mathrm{ml}^{-1}\right.$ of specific radioactivity $100 \mu \mathrm{Ci} \cdot \mathrm{mmol}^{-1}$ from New England Nuclear, Dreieich, FRG) has been added, followed by $15 \mathrm{~min}$ open circuit perfusion with radioacetate-free medium. Weight increases in the perfused blocks of ca. $30 \%$ indicated satisfactory perfusion.

Cytoplasmic extracts were prepared from the frozen tissue by crushing it and extracting the resultant coarse powder while still frozen with a small volume of $0.4 \mathrm{M} \mathrm{NaCl}$ approximately equal to the tissue weight; they were then centrifuged at $10,000 \mathrm{rpm}$ for 30 min to remove larger particles; the supernatant so obtained was sampled [ $3 \pm 1(4) \mathrm{ml}]$ and the remainder [ $34 \pm 2(4)$ ml equivalent to $54 \pm 6(4) \mathrm{g}$ of tissue] applied to a sucrose- $\mathrm{NaCl}$ density gradient formed ${ }^{6}$ in a Beckman Ti-60 330-ml capacity zonal rotor. After centrifuging at $50,000 \mathrm{rpm}$ for $3 \mathrm{~h}$, the gradient was pumped out and collected in $5 \mathrm{ml}$ fractions. The zonal centrifugation procedure has been fully described $d^{6,38}$ and was carried out in the cold $\left(4^{\circ} \mathrm{C}\right)$. The gradient became hyperosomotic when its refractive index (RI) exceeded 1.3700

\section{Analytical methods}

Acetylcholine was assayed on small strips of the dorsal muscle of the leech as previously described ${ }^{37}$ after having been released from particulate material in cytoplasmic extracts and density gradient fractions and stabilized by bringing samples of the fractions to $\mathrm{pH}$ 4.0. Samples were stored at $-20^{\circ} \mathrm{C}$ until assayed.

$A T P$ was determined in deproteinized supernatants of samples of subcellular fractions by a modification ${ }^{10}$ of the luciferin-luciferase bioluminescence method ${ }^{33}$. The distribution of ATP in the gradients closely followed acetylcholine and its assay was done mainly as a rapid means for locating the fractions containing synaptic vesicles before assaying for acetylcholine and other markers.

Protein was determined by the bicinchonitric acid method ${ }^{29}$ using a reagent kit supplied by the Pierce Chemical Co (Rockford IL, USA).

Sucrose in density gradients was determined by measuring the RI in an Abbé refractometer. To facilitate comparisons between different zonal runs in which gradient profiles may differ slightly, analyses of gradient components were plotted as a function of RI, not of fraction number. From the RI, the sucrose concentration and density of fractions may be deduced from tables. In the main part of the gradient the RI rose at the rate of $0.0006-0.0008$ units per 5-ml fraction, but increased more rapidly above RI 1.3600 .

Comparisons between runs was also facilitated by the normalization of results in which the content of a component in each fraction is expressed as a percentage of the total amount of the component recovered from the gradient. The efficiency of the separation was checked by calculating the recovery of each component as a percentage of the amount of it applied to the density gradient at the commencement of each run. Recoveries were close to $100 \%$.

\section{Evaluation of synaptic vesicle numbers}

Density gradient fractions. Vesicles were counted in fractions by a modification of a previously described method ${ }^{27}$. They were fixed by treating a $1-\mathrm{ml}$ sample of each fraction immediately after collection with $1 \mathrm{ml}$ of cold $\left(4^{\circ} \mathrm{C}\right) 2 \%$ glutaraldehyde in $0.4 \mathrm{M}$ sodium cacodylate buffer, $\mathrm{pH} 7.4$ (SCB). After varying periods of storage, during which vesicles appeared to be stable, fractions were further processed for electron microscopy by adding $2 \mathrm{ml}$ of SCB and $1 \mathrm{ml}$ of $4 \%$ aqueous $\mathrm{OsO}_{4}$. After $1 \mathrm{~h}$, the mixture was centrifuged for $1 \mathrm{~h}$ at $100,000 \mathrm{~g}_{\text {av }}$ in a Beckman SW 50 rotor; the pellet so obtained was gently resuspended in $1 \mathrm{ml}$ of SCB using a hand operated Dounce homogenizer with a loosely fitting pestle and the particulate material in the suspension collected by filtration on a 5 -mm diameter Millipore filter of $0.45 \mu \mathrm{m}$ pore size. The filter was washed with $1 \mathrm{ml} \mathrm{SCB}$, suction stopped and $0.1 \mathrm{ml}$ of $3 \%$ liquid agar poured over the filter to form a protective film. On cooling, the filter was detached, bisected and the two halves processed for electron microscopy by dehydration in ethanol and embedding in Epon 812. Blocks were trimmed so as to provide a $3 \mathrm{~mm}$ length of a vertical section through the vesicle pellet and a $1 \mu \mathrm{m}$ section was cut and stained with Toluidine blue for a light microscopic determination of pellet thickness. This was done by taking the mean of 10 measurements along the length of the section with an ocular micrometer at a magnification factor of 400 .

Thin (120 $\mathrm{nm}$ thick) sections were then cut from the same piece for electron microscopy. They were mounted without support on 150 mesh grids and counterstained with uranium and lead. A series of 5-10 micrographs were made of randomly selected areas along the $3 \mathrm{~mm}$ length of the pellet section at a magnification of 24,000 and prints were made from these with an additonal 3-fold enlargement. Profiles were counted and the number of vesicles per unit volume deduced by correcting for vesicle centres falling outside the section ${ }^{1}$. The multiplication factor is such that one vesicle per print corresponds to $10^{8}$ vesicles $\cdot \mathrm{ml}^{-1}$ of fraction. Samples containing much protein were difficult to embed and cut; for this reason no attempt was made to count vesicles in the cytoplasmic extract or to evaluate vesicle recoveries.

Tissues. The number of vesicles present within nerve terminals in intact tissue was counted in electron micrographs of sections cut vertically through stacks of electrocytes. The relative volume of nerve terminals was calculated from measurements of the fraction of the area of micrographs occupied by nerve terminal profiles.

\section{RESULTS}

Morphological and biochemical characterization of gradient fractions

All sealed vesicular profiles were counted independently of size or shape. The mean yield of isolated vesicles $\left(\times 10^{-13}\right)$ per run was $1.58 \pm 0.23$ or $0.32 \pm 0.09$ 
per pg of tissue. Few or no vesicles were present in the early fractions; these were rich in soluble cytoplasmic proteins $^{38}$ (Fig. 1) and somewhat difficult to section. Vesicles were first detected at about fraction $18(90 \mathrm{ml}$; RI, 1.350) and peaked (Fig. 1, peak I and Table I) at RI $1.3584 \pm 0.0002(4)$, consistently one or two fractions beyond the peak of acetylcholine at RI $1.3577 \pm 0.0006$. The profiles in these fractions were identical in morphology to those of the main population of synaptic vesicles in nerve terminals; the mean profile diameter (Fig. 1, insert) was close to those previously reported ${ }^{15-18,27}$. 28.41,42, using transmission electron microscopy and ultrathin sections ${ }^{16,17,27,28,41,42}$ or negative staining ${ }^{28}$, or a non-invasive biophysical method ${ }^{15.16}$. There were, in this region of the gradient, few profiles over $120 \mathrm{~nm}$ in diameter (Fig. 1, insert). Vesicles in small numbers were found all the way down the gradient and formed two smaller peaks (Fig. 1, II and III) at about RI 1.3670 and 1.3780 respectively. These denser fractions contained vesicular profiles up to $1400 \mathrm{~nm}$ in diameter as well as non-vesicular contamination. As previously noted ${ }^{7}$, peak II coincided approximately with a peak of thiamine pyrophosphatase activity (Table I) and may thus have contained vesicles associated with, or derived from Golgi membranes, since thiamine pyrophosphatase is a recognized marker for such membranes. Peak III contained dense-cored vesicles in addition to those with electrontranslucent cores; the mean profile diameter was $115 \mathrm{~nm}$ (Fig. 1, insert) and the peak coincided (Table I) with one of a polypeptide immunochemically resembling porcine vasoactive intestinal polypeptide (VIP) ${ }^{2.3}$. This peptide is known to be present, in many tissues, within cholinergic neurons and to be packaged in large dense-cored vesicles $^{3.5}$.

Synaptophysin (also known as p38), a recognized marker for synaptic vesicles ${ }^{20.39}$ and probably for other storage particles as well ${ }^{3}$, was also distributed right down the gradient; it formed two peaks, one coinciding with peak I of synaptic vesicles and the acetylcholine peak, the other approximately with peak III and the peak of

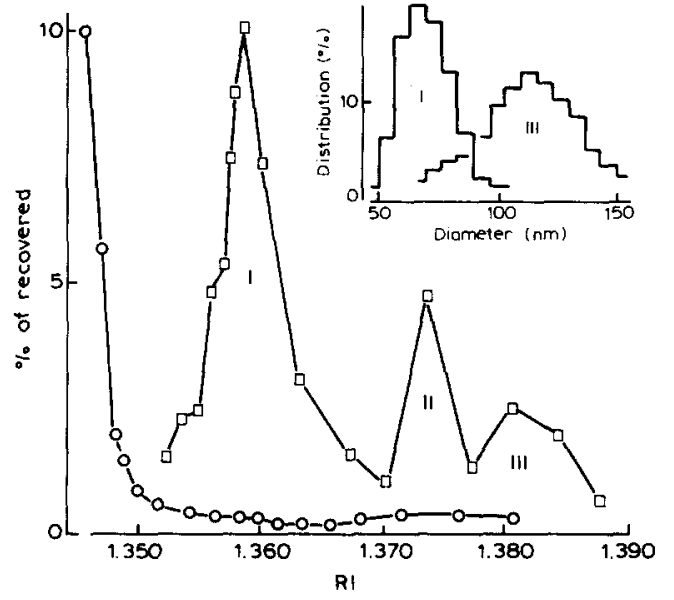

Fig. 1 Distribution in a zonal density gradient of (squares) vesicle numbers and (circles) protein. Insert: distribution of vesicle diameters in peak fractions of peaks I and III (from Agoston et al. ${ }^{7}$ ).

electromotor VIP (Table I). Although it was present in the region of vesicle peak II, it formed no definite peak in that region of the gradient.

\section{The distribution of acetylcholine in the gradient}

Endogenous acetylcholine. Acetylcholine formed, as expected, a single peak in the gradient with an RI of $1.3577 \pm 0.0006(4)$; the slightly lower value $[1.3552 \pm$ 0.0002 (16)] reported earlier ${ }^{6}$ may have been due to a slightly lower operating temperature in the earlier runs. In some experiments (Fig. 2a,c) the peak was sharp and symmetrical; in others (Fig. 2b,d), a more or less pronounced shoulder could be seen on the dense side of the peak. When the number of acetylcholine molecules per vesicle (the molecular acetylcholine content) was calculated for each fraction by multiplying its molar acetylcholine content by Avogadro's number and dividing by the number of vesicles per litre, it became clear that the synaptic vesicles within peak I could indeed be grouped into at least 3 subpopulations or pools: a small number of light vesicles with a relatively low acetylcholine content (Fig. 2, $\mathrm{V}_{0}$ ), a main population of vesicles

TABLE I

Biochemical characterization of vesicle fractions prepared by centrifugal separation in a sucrose-NaCl density gradient

\begin{tabular}{|c|c|c|c|c|}
\hline \multirow[t]{2}{*}{ Parameter } & \multirow[t]{2}{*}{ Marker for } & \multicolumn{3}{|c|}{ Mean RIs of peaks of vesicle distribution } \\
\hline & & $I$ & II & $I I I$ \\
\hline Vesicles $^{\mathbf{a}}$ & & $1.3584 \pm 0.0002(4)$ & $1.3670 \pm 0.0027(4)$ & $1.3783 \pm 0.0023(2)$ \\
\hline Acetylcholine ${ }^{\mathbf{a}}$ & synaptic vesicles & $1.3577 \pm 0.0006(4)$ & - & - \\
\hline TPPase $^{b}$ & Golgi membranes & - & 1.3740 & - \\
\hline VIP-like immunoreactivity ${ }^{\mathbf{b}}$ & dense-cored vesicles & - & - & 1.3783 \\
\hline Synaptophysin ${ }^{\mathrm{b}}$ & storage particles & 1.3605 & - & 1.3783 \\
\hline
\end{tabular}

\footnotetext{
Values are means \pm S.E.M. of 4 or \pm range of 2 experiments in which measurements were extended to denser regions of the gradient

b Results of Agoston et al. ${ }^{7}$ converted to RI values.
} 
highly charged with acetylcholine (Fig. 2, $V_{1}$ ) and a population of denser vesicles (Fig. $2, \mathrm{~V}_{2}$ ), again with a lower acetylcholine content. The main $V_{1}$ subpopulation had a mean molecular acetylcholine content in satisfactory agreement with, though somewhat higher than that reported earlier ${ }^{27}$ for the peak acetylcholine fractions. Since the fractions obtained in the earlier work had been worked up promptly for electron microscopy, this agree- ment suggests that some loss of vesicles may have occurred during storage and subsequent processing for electron microscopy but if so, this could not have been serious.

Vesicle counts did not, in general, show the smooth progression from fraction to fraction seen with acetylcholine and other parameters and there appeared to be a random fluctuation superimposed upon their distribu-
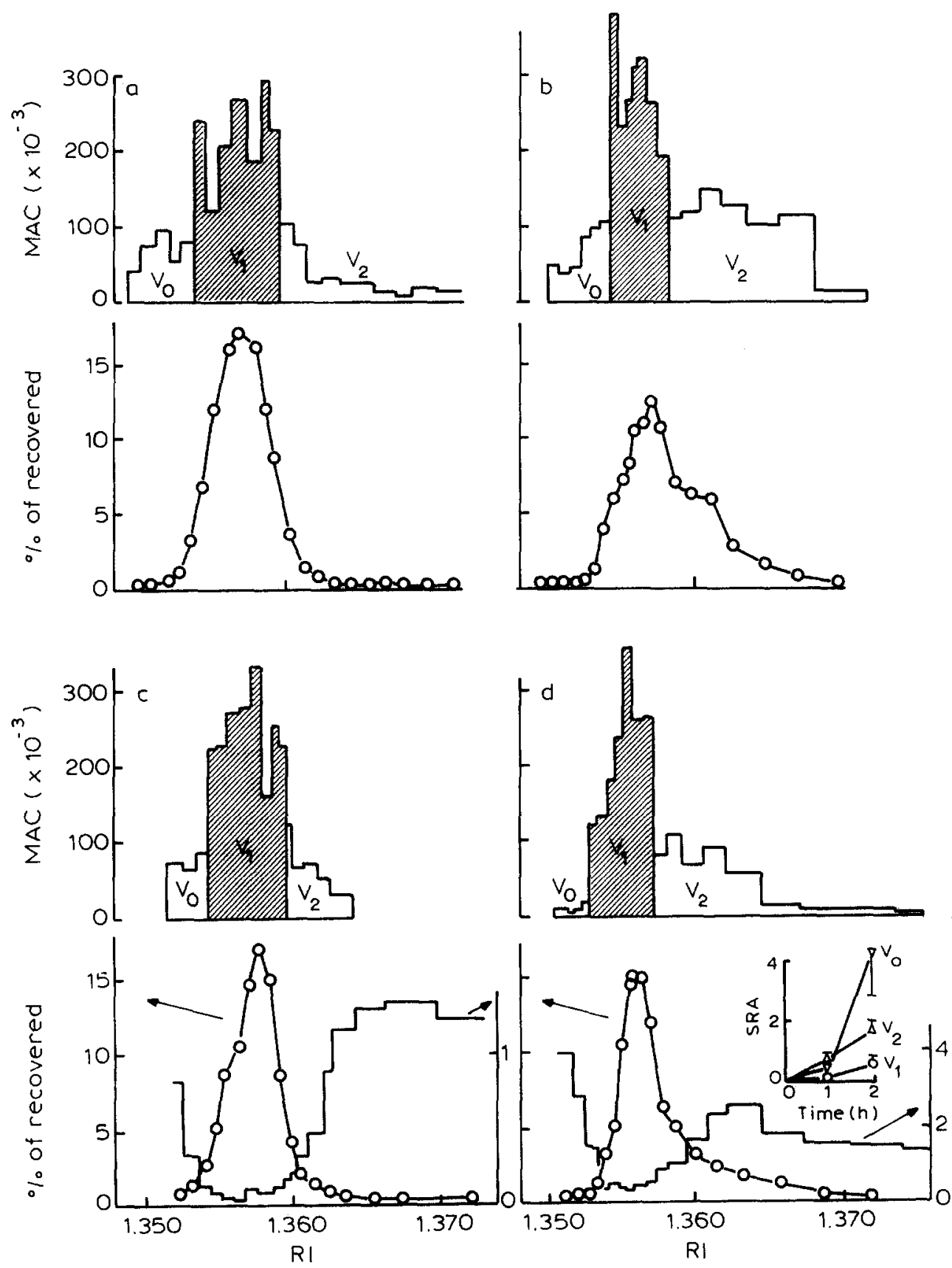

Fig. 2. Distribution in zonal density gradients of vesicular acetylcholine (circles) after extraction of vesicles from blocks (a,b) frozen immediately after dissection, or (c,d) after perfusion with $\left[{ }^{3} \mathrm{H}\right]$ acetate for (c) 1, (d) $2 \mathrm{~h}$. The upper histograms in a-d show the molecular acetylcholine content (MAC) of the vesicles in each fraction, the lower ones (c,d only) the specific radioactivity (SRA) of each fraction. Note that the scale of SRA in $c$ is twice that in $d$. The insert in d shows the mean SRA of each vesicle subpopulation as a function of time. The results permit classification of the vesicles into 3 subfractions differing in density, acetylcholine content and ability to incorporate newly synthesized transmitter. 
TABLE II

Acetylcholine content of subpopulations of synaptic vesicles isolated from unstimulated electric tissue

\begin{tabular}{|c|c|c|c|c|c|c|c|}
\hline \multirow[t]{3}{*}{ Block } & \multirow{3}{*}{$\begin{array}{l}\text { Perfusion } \\
\text { time }(h)\end{array}$} & \multicolumn{6}{|c|}{ Percentage (a) and molecular content $\left(\times 10^{-3}\right)(b)$ of acetylcholine in synaptic vesicle subpopulations } \\
\hline & & \multicolumn{2}{|l|}{$V_{0}$} & \multicolumn{2}{|l|}{$V_{1}$} & \multicolumn{2}{|l|}{$\underline{V_{2}}$} \\
\hline & & (a) & (b) & (a) & (b) & (a) & (b) \\
\hline a & 0 & 5 & $70 \pm 9(5)$ & 88 & $223 \pm 22(7)$ & 7 & $36 \pm 11(9)$ \\
\hline$c$ & 1 & 5 & $76 \pm 7(3)$ & 86 & $245 \pm 19(8)$ & 9 & $73 \pm 19(4)$ \\
\hline b & 0 & 7 & $73 \pm 11(6)$ & 67 & $285 \pm 23(7)$ & 26 & $127 \pm 6(7)$ \\
\hline d & 2 & 1 & $13 \pm 3(4)$ & 79 & $229 \pm 28(8)$ & 20 & $81 \pm 8(5)$ \\
\hline & - & $4.5 \pm 1.7$ & $58 \pm 15$ & $80 \pm 5$ & $246 \pm 14$ & $16 \pm 5$ & $79 \pm 19$ \\
\hline \multicolumn{2}{|c|}{$\begin{array}{l}\text { Ohsawa et al. }{ }^{27} \\
\text { (acetylcholine peak fractions) }\end{array}$} & - & - & - & $260 \pm 58(5)$ & - & - \\
\hline
\end{tabular}

tion in the gradient. The cause for this was not traced but was not due to counting errors, since independent counts made on any one set of electron micrographs usually agreed to within $10 \%$. The fluctuations may have been caused by any or all of the following: random losses during processing, inhomogeneities within vesicle pellets and the smallness of the samples of the pellets taken for electron microscopy. Because of these fluctuations in the observed concentration of vesicles in the fractions, there was a fairly large variance in the mean molecular acetylcholine content within each population; however, differences between the $V_{1}$ and $V_{2}$ and between the $V_{1}$ and $V_{0}$ subpopulations were statistically significant $(p<0.01)$ (Table II).

Newly synthesized acetylcholine. In experiments in which radioactive acetate had been perfused, radioactive (i.e. newly synthesized) acetylcholine was taken up by all the synaptic vesicle fractions; however, when the specific radioactivity of the vesicular acetylcholine in each fraction was calculated, it became clear that the acetylcholine of both the $V_{0}$ and $V_{2}$ vesicles was considerable more radioactive than that of the fully charged $V_{1}$ vesicles (Fig. $2 c, d)$. As expected, the amount of labelling of each fraction was greater after $2 \mathrm{~h}$ of perfusion than after $1 \mathrm{~h}$ (Fig. 2d, insert). Recent evidence suggests that vesicular acetylcholine uptake is regulated by the vesicles' internal content of acetylcholine and that uptake into replete vesicles is severely reduced ${ }^{19,26,32}$.

\section{DISCUSSION}

\section{Characterization of synaptic vesicles}

The $V_{1}$ pool. The molecular acetylcholine content of the vesicles in the main portion of the acetylcholine peak is very close to that found in earlier work (Table II). Some very high values in individual fractions could be due to adventitious losses of vesicles during storage or preparation for electron microscopy. Stages at which losses or errors could have occurred include: breakdown during handling of vesicles rendered fragile by fixation, inhomogeneities in vesicle pellets inadequately revealed by the very small samples represented by the ultrathin sections viewed in the electron microscope, and inadequacies in the stereological routines which were a little different from those previously used ${ }^{27}$. It proved impossible to control all these factors, but, even assuming a percentage or fixed loss from each fraction, the statistical differences between $V_{1}$ and the two other pools would remain. The osmotic load of reserve vesicles has been estimated by a non-invasive biophysical technique ${ }^{15}$ to be 0.21 aosmol per vesicle; this is consistent with the previously observed data ${ }^{27}$ on the basis of an assumed activity coefficient of 0.65 , a not unreasonable value for the concentrated solution of acetylcholine and ATP present in the vesicle core.

Recycling vesicles are present in unstimulated tissue. It seems clear, then, that two $\left(V_{1}\right.$ and $\left.V_{2}\right)$ of the 3 subpopulations of synaptic vesicles here demonstrated to be present in resting blocks of electric organ tissue can be identified with the reserve $\left(\mathrm{VP}_{1}\right)$ and recycling $\left(\mathrm{VP}_{2}\right)$ vesicle pools previously shown to be present in stimulated tissue $^{6,18,41}$. Vesicles isolated from resting tissue, though apparently homogeneous on the basis of the unimodal distribution of acetylcholine in the gradient and the relative symmetry of its peak, always show heterogeneity with respect to the incorporation of radiolabelled acetylcholine when labelled transmitter precursors are perfused $^{41,44}$. This takes the form of a rising specific radioactivity on the descending, dense limb of the acetylcholine peak and has been held to show that recycling vesicles are also present in resting tissue. These may result from spontaneous quantized transmitter release (electromotor nerve terminals generate miniature postsynaptic potentials $^{1,12,30}$ ), occasional discharges by 
TABLE III

Proportion of subpopulations of synaptic vesicles in the total population isolated

\begin{tabular}{lllll}
\hline Block & \multicolumn{2}{l}{ Percentage of synaptic vesicles in } & $\begin{array}{l}100 V_{2} \\
\left(V_{1}+V_{2}\right)\end{array}$ \\
\cline { 2 - 3 } & $V_{0}$ & $V_{1}$ & $V_{2}$ & \\
\hline a & 11 & 64 & 25 & 28 \\
c & 12 & 65 & 23 & 26 \\
b & 16 & 46 & 38 & 45 \\
d & 12 & 37 & 51 & 58 \\
Mean \pm S.E.M. & $13 \pm 1$ & $53 \pm 7$ & $34 \pm 6$ & $39 \pm 7$ \\
\hline
\end{tabular}

the living fish, or adventitious discharges during dissection, the reality of which can be testified to by anyone who has carried out dissection with bare hands! Heterogeneity has also been demonstrated with respect to acetylcholine uptake in vitro ${ }^{32}$.

Although $V_{2}$ vesicles could be readily distinguished from $V_{1}$ vesicles on the basis of their lower transmitter content and greater density, they represent a dynamic population with a gradient of densities and transmitter contents. Though the gradient failed to resolve subclasses within the $V_{2}$ fraction there is sufficient heterogeneity with respect to acetylcholine content to accommodate the phenomenon of subminiature postsynaptic potentials, if indeed these occur at the electromotor synapse (the evidence is equivocal; compare refs. 22 and 24).

One might expect that perfusion would give time for $\mathrm{V}_{2}$ vesicles to become replete and join the $V_{1}$ pool as has been demonstrated for stimulated tissue ${ }^{6,18,41}$; this, however, is a slow process ${ }^{40}$ and takes up to $16 \mathrm{~h}$ to be completed $^{6}$. In the present series of experiments perfusion was carried out only for sufficient time to allow for labelling of the recycling pool.

That the size of this pool in resting tissue may be largely determined by the amount of adventitious stimulation the latter receives during the experiment seems to be borne out in the present series of experiments by the variations seen in the partition of synaptic vesicles between the $V_{1}$ and $V_{2}$ pools in identically handled blocks (Table III). The proportion of the $V_{1}+V_{2}$ vesicles in the $\mathrm{V}_{2}$ pool varied from 26 to $58 \%$. The size of the $\mathrm{V}_{2}$ pool was not so evident from the distribution of acetylcholine between the two pools (Table II), since only $7-26 \%$ of the transmitter is recovered in it and only at the upper end of this range is its presence revealed by a shoulder in the otherwise symmetrical acetylcholine peak.

Significance of the $V_{0}$ pool. A low-density vesicle fraction isolated from electromotor nerves but lacking acetylcholine was demonstrated $d^{21,32}$ in pulse-labelling

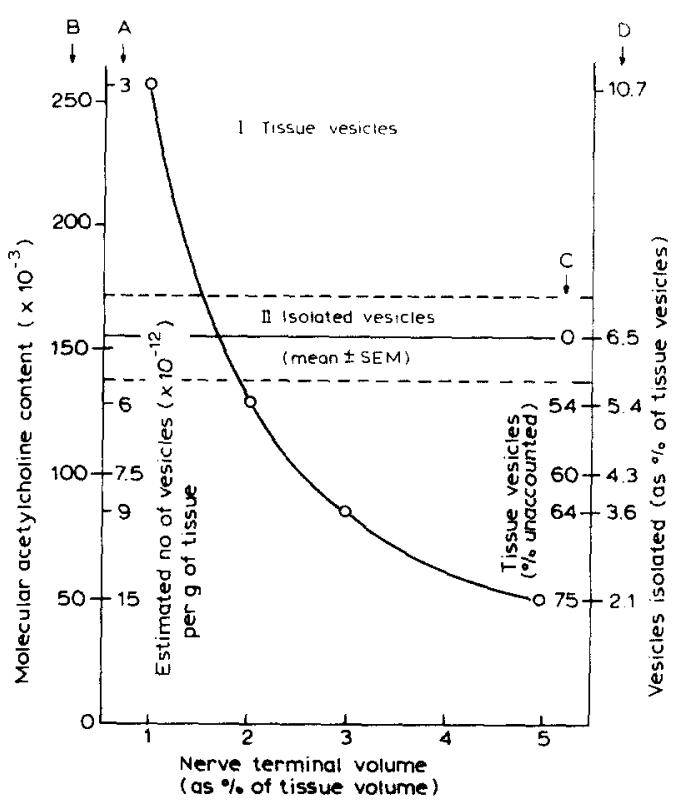

Fig. 3. Curve I shows (on ordinate scale A) the estimated no of vesicles per $\mathrm{g}$ of tissue and (scale $\mathrm{B}$ ) the mean molecular acetylcholine content of tissue vesicles based on an estimate of $3 \times$ $10^{14}$ vesicles $\cdot \mathrm{ml}^{-1}$ of nerve terminal and a range of assumptions (abscissae) as to the vol-\% of the tissue occupied by nerve terminals. Line II is the mean molecular acetylcholine content of isolated vesicles. It can be seen that if nerve terminals occupy $1.7 \pm 0.2 \%$ of the tissue (a not unreasonable assumption) there are no tissue vesicles unaccounted for (scale $C$ ) in the sample isolated, which represents (scale D) $6.5 \%$ of the tissue vesicles. The calculations are based on a mean tissue acetylcholine content of $1.28 \mu \mathrm{mol}(7.68 \times$ $10^{18}$ molecules) $\mathrm{g}^{-1}$ (or ml $\mathrm{m}^{-1}$ ) of tissue and a yield of isolated vesicles of $3.2 \times 10^{11}$ per $g$ of tissue.

experiments with ${ }^{35} \mathrm{~S}$ to be also present in the electromotor nerve terminals where it took up acetylcholine and, in some hours, acquired the density of replete $\left(\mathrm{VP}_{1}\right)$ vesicles. Partial confirmation of these findings came from the isolation from electromotor axons of vesicles of similar density and very low acetylcholine and ATP content ${ }^{7}$. The $V_{0}$ vesicles in the present study had a similar density; they also (Fig. 1c,d) avidly took up newly synthesized acetylcholine in situ, and are thus probably to be identified with the $\mathrm{VP}_{0}$ pool of axonal synaptic vesicles that have recently arrived in the nerve terminal. The contribution of these to the total synaptic vesicular pool is however quite low (Table III, $13 \pm 1(4) \%$ ) and less than $5 \%$ of the vesicular acetylcholine is on average associated with them (Table II).

Vesicle diameters. In previous work ${ }^{18,40-42}$ the mean diameter of vesicles of the pooled $\mathrm{VP}_{2}$ fractions as deduced from stereological transformation of the distribution of vesicle profile diameters has been found to be about $25 \%$ less than that of similarly pooled $\mathrm{VP}_{1}$ vesicles. This is consistent with a biophysical model of the vesicle ${ }^{15}$ deduced from measurements of the density and water 
content of vesicles of different degrees of osmotic loading, in which it is regarded as an osmometer with an aqueous core containing dissolved solutes (acetylcholine and ATP) and surrounded by a membrane selectively permeable to water. No repeat comparisons of vesicle diameters were made in the present work since (a) the density gradient was not iso-osmotic throughout its length and (b) in contrast to previous work vesicles were kept in fixative sometimes for a considerable time before processing (see Methods) and would be expected to have equilibrated with the suspension medium. Under these conditions $^{14}$ it was felt that any attempt to detect small differences in diameter between fractions would be unlikely to succeed.

\section{Are isolated vesicles representative of the tissue vesicle} population?

The isolation of an organelle in a high state of purity and homogeneity, like that of an enzyme or other protein, entails losses, and most isolation procedures are a compromise between yield and purity. Because of the high collagen content of the electric organ, its comminution requires vigorous methods, such as the freezing and crushing procedure originated by Whittaker et al. ${ }^{38}$. About $50 \%$ of the total tissue acetylcholine remains bound (presumably vesicular); of the 50\% lost, about $40 \%$ has been shown, by a non-invasive technique $e^{35}$ to be free, cytoplasmic acetylcholine. In the subsequent fractionation there are losses due to leakage of acetylcholine from the bound state which are revealed when recoveries fall below $100 \%$ and losses of bound acetylcholine along with discarded coarse tissue fragments in the $\mathrm{P}_{12}$ fraction. Just under $50 \%$ of the bound acetylcholine was originally recovered in the cytoplasmic $\left(S_{12}\right)$ extract ${ }^{38}$. In later work in which, in order to reduce the amount of soluble cytoplasmic protein extracted, the ratio of the amount of crushed tissue extracted to the volume of extraction medium was increased and the temperature of extraction lowered, the proportion of bound acetylcholine was lower $(27 \%)^{41}$. Recoveries from the zonal gradient are usually satisfactory ( $98 \pm 3 \%$ in the present series). Thus, although the percentage of total tissue acetylcholine or of the estimated amount of tissue vesicular acetylcholine recovered from the gradient is quite low, losses during fractionation can be precisely accounted for and there is no reason to suppose that what is isolated is not a fully representative sample of the original vesicle population. This proposition may in principle be tested in the following way.

The estimate of the tissue content of vesicular acetylcholine made by a non-invasive technique ${ }^{35}$ when divided by the estimated number of vesicles in unit weight of tissue gives us the amount of acetylcholine per vesicle.
This may then be compared to the average acetylcholine content per vesicle of the isolated vesicles.

Unfortunately it is not a trivial matter to determine the number of vesicles per unit weight of tissue, since this has to be done by stereological methods from conventional electron micrographs and it is difficult to sample the tissue as a whole or to control for the amount of shrinkage which occurs when the tissue is prepared for electron microscopy. Using the procedure described in Materials and Methods I obtained the value of $303 \pm 30$ (11) vesicles $\mu \mathrm{m}^{-3}$ for nerve terminals. This is in the range obtained by Fox et al. ${ }^{13}$ (300-325). In sections through stacks of electrocytes, nerve terminals occupy about $5 \%$ of the volume of the fixed, embedded and sectioned tissue but this does not take into account the morphology of the edges of the electrocytes, where innervation is reduced, the proportion of the volume of the tissue taken up by the nerve trunks, the blood vessels, and the extracellular space between the electrocyte stacks, nor the effect of tissue shrinkage during dehydration and embedding. Thus a value of half or less of this might be more reasonable. Fig. 3 gives (curve I, scale A) the number of vesicles $\cdot \mathrm{ml}^{-1}$ of tissue for a range of assumptions (abscissae) concerning the proportion of the whole tissue occupied by nerve terminals, based on the estimate of $3 \times 10^{14}$ vesicles $\cdot \mathrm{ml}^{-1}$ of nerve terminal.

The acetylcholine content of the tissue samples from which the electron micrographs were made is not known and published values vary over quite a wide range. However, Weiler et al. ${ }^{35}$, using gas chromatography, obtained a mean value of $1.64 \mu \mathrm{mol} \cdot \mathrm{g}^{-1}$ of which $22 \%$ was estimated to be cytoplasmic by a non-invasive technique. This gives for vesicular acetylcholine, 1.28 $\mu \mathrm{mol} \cdot \mathrm{g}^{-1}$ of tissue or $7.68 \times 10^{17}$ molecules $\cdot \mathrm{g}^{-1}$. The estimated mean molecular acetylcholine content per vesicle thus ranges (Fig. 3, scale B) from 51,000 to 256,000 depending on the assumptions made. This may be compared to $155,000 \pm 17,000(4)$ as the mean value for the isolated synaptic vesicles (Fig. 3, line II). Clearly this leaves no room for large numbers of tissue vesicles that remain unaccounted for by the sample isolated as has been maintained by Fox et al. ${ }^{14}$, even though this may represent only $2-10 \%$ of the tissue vesicle population (scale D), again depending on the assumptions made. In scale $C$, the proportion of empty vesicles unaccounted for in the sample isolated is calculated. It ranges from $75 \%$ of the tissue vesicle population if $5 \%$ of the tissue is nerve terminals to zero if it is $1.7 \%$. If it is only $1 \%$, we should have to assume that all the vesicles in the tissue are fully charged with transmitter to the level observed in the $V_{1}$ fraction and that a considerable proportion of them had lost part or all of their acetylcholine during isolation, which is not borne out by the recoveries during fraction- 
ation. It thus seems implausible that there is a class of empty vesicles in the tissue which is selectively destroyed by freezing, extraction etc., or selectively left behind in the discarded fractions and so not represented in the isolated vesicles as proposed by Fox et al. ${ }^{14}$.

What these calculations also serve to show is the difficulty of making quantitative estimates based on the small samples provided by the highly invasive technique of conventional electron microscopy of whole tissue.

\section{REFERENCES}

1 Abercrombie, M., Estimation of nuclear population from microtomic sections, Anat. Rec., 94 (1946) 239-247.

2 Agoston, D.V. and Conlon, J.M., Presence of vasoactive intestinal polypeptide-like immunoreactivity in the cholinergic electromotor system of Torpedo marmorata, J. Neurochem., 47 (1986) 445-453.

3 Agoston, D.V. and Whittaker, V.P., Characterization, by size, density, osmotic fragility and immunoaffinity, of acetylcholineand vasoactive intestinal polypeptide-containing storage particles from myenteric neurones of the guinea pig, J. Neurochem., 52 (1989) 1474-1480.

4 Agoston, D.V., Kosh, J.W., Lisziewicz, J. and Whittaker, V.P., Separation of recycling and reserve synaptic vesicles from the cholinergic nerve terminals of the myenteric plexus of guinea pig ileum, J. Neurochem., 44 (1985) 299-305.

5 Agoston, D.V., Ballmann, M., Conlon, J.M., Dowe, G.H.C. and Whittaker, V.P., Isolation of neuropeptide-containing vesicles from the guinea pig ileum, J. Neurochem., 45 (1985) 398-406.

6 Agoston, D.V., Dowe, G.H.C., Fiedler, W., Giompres, P.E., Roed, I.S., Walker, J.H., Whittaker, V.P. and Yamaguchi, T., The use of synaptic vesicle proteoglycan as a stable marker in kinetic studies of vesicle recycling, $J$. Neurochem., 47 (1986) 1580-1592.

7 Agoston, D.V., Dowe, G.H.C. and Whittaker, V.P., Isolation and characterization of secretory granules storing a vasoactive intestinal polypeptide-like peptide in Torpedo cholinergic electromotor neurones, J. Neurochem., 52 (1989) 1729-1740.

8 Barker, L.A., Dowdall, M.J. and Whittaker, V.P., Choline metabolism in the cerebral cortex of guinea pig, Biochem. J., 130 (1972) 1063-1075.

9 Chakrin, L.W., Marchbanks, R.M., Mitchell, J.F. and Whittaker, V.P., Origin of acetylcholine released from the surface of the cortex, J. Neurochem., 19 (1972) 2727-2736.

10 Dowdall, M.J., Boyne, A.F. and Whittaker, V.P., Adenosine triphosphate: a constituent of cholinergic synaptic vesicles, Biochem. J., 140 (1974) 1-12.

11 Dunant, Y, and Muller, D., Quantal release of acetylcholine evoked by focal depolarization at the Torpedo nerve-electroplaque junction, J. Physiol. (Lond.), 379 (1986) 461-478.

12 Erdelyi, L. and Krenz, W.-D., Electrophysiological aspects of synaptic transmission at the electromotor junction of Torpedo marmorata, Comp. Biochem. Physiol., 79A(1984) 505-511.

13 Fox, G.Q., Kriebel, M.E. and Kötting, D., Synaptic vesicle classes in Torpedo and skate electric organ and muscle. In $\mathrm{H}$. Zimmermann (Ed.), Cellular and Molecular Basis of Synaptic Transmission, NATO ASI Series, Vol. H21 Springer, Berlin/ Heidelberg (1988) pp. 83-95.

14 Fox, G.Q., Kötting, D. and Dowe, G.H.C., A morphometric analysis of Torpedo synaptic vesicles isolated by iso-osmotic sucrose gradient separation, Brain Research, 498 (1989) 279288.

15 Giompres, P.E. and Whittaker, V.P., The density and free water
Acknowledgements. I am grateful to Mr. G.H.C. Dowe who undertook the tedious tasks of preparing the centrifugal density gradient fractions for electron microscopy and counting the vesicle profiles in electron micrographs of vesicle pellets. Section cutting, screening and computations were carried out by Herrn D. Kötting and $\mathrm{Dr}$. G.Q. Fox in the electron microscope laboratory formerly part of the now defunct Abteilung Neurochemie of the MaxPlanck-Institut für Biophysikalische Chemie. To these ex-colleagues I express my thanks. The participation of Dr. P.E. Giompres in the radiolabelling experiments and of Dr. D.V. Agoston in the vesicle diameter distribution study is gratefully acknowledged. The work was in part supported by the Deutsche Forschungsgemeinschaft (Grant Wh 1/4-2)

of cholinergic synaptic vesicles as a function of osmotic pressure, Biochim. Biophys. Acta, 882 (1986) 398-409.

16 Giompres, P.E., Morris, S.J. and Whittaker, V.P., The water spaces in cholinergic synaptic vesicles from Torpedo measured by changes in density induced by permeating substances, Neuroscience, 6 (1981) 757-763.

17 Giompres, P.E., Zimmermann, H. and Whittaker, V.P., Purification of small dense vesicles from stimulated Torpedo electric tissue by glass bead column chromatography, Neuroscience, 6 (1981) $765-774$.

18 Giompres, P.E., Zimmermann, H. and Whittaker, V.P., Changes in the biochemical and biophysical parameters of cholinergic synaptic vesicles on transmitter release and during a subsequent period of rest, Neuroscience, 6 (1981) 775-785.

19 Gracz, L.M., Wang, W.-C. and Parsons, S.M., Cholinergic synaptic vesicle heterogeneity: evidence for regulation of acetylcholine transport, Biochemistry, 27 (1988) 5268-5274.

20 Jahn, R., Schiebler, W., Ouimet, C. and Greengard, P., A 38,000-dalton membrane protein (p38) present in synaptic vesicles, Proc. Natl. Acad. Sci. U.S.A., 82 (1985) 4137-4141.

21 Kiene, M.L. and Stadler, H., Synaptic vesicles in electromotoneurones, I. Axonal transport, site of transmitter uptake and processing of a core proteoglycan during maturation, EMBO J., 6 (1987) 2209-2215.

22 Kriebel, M.E., Fox, G.Q. and Kötting, D., Effect of nerve stimulation, $\mathrm{K}^{+}$saline and hypertonic saline on classes of quanta, quantal content and synaptic vesicle size distribution of Torpedo electric organ. In $\mathrm{H}$. Zimmermann (Ed.), Cellular and Molecular Basis of Synaptic Transmission, NATO ASI Series Vol. H21, Springer, Berlin/Heidelberg, 1988, pp. 97-120.

23 Lee, D.A. and Witzemann, V., Photoaffinity labeling of a synaptic vesicle specific nucleotide transport system from Torpedo marmorata, Biochemistry, 22 (1983) 6123-6130.

24 Muller, D. and Dunant, Y., Spontaneous quantal and subquantal transmitter release at the Torpedo nerve-electroplaque junction, Neuroscience, 20 (1987) 911-921.

25 Nagy, A., Baker, R.R., Morris, S.J. and Whittaker, V.P., The preparation and characterization of synaptic vesicles of high purity, Brain Research, 109 (1976) 285-309.

26 Noremberg, K. and Parsons, S.M., Regulation of the vesamicol receptor in cholinergic synaptic vesicles by acetylcholine and an endogenous factor, J. Neurochem., 52 (1989) 913-920.

27 Ohsawa, K., Dowe, G.H.C., Morris, S.J. and Whittaker, V.P., The lipid and protein content of cholinergic synaptic vesicles from the electric organ of Torpedo marmorata purified to constant composition: implications for vesicle structure, Brain Research, 161 (1979) 447-457.

28 Sheridan, M.N., Whittaker, V.P. and Israël, M., The subcellular fractionation of the electric organ of Torpedo, Z. Zellforsch., 74 (1965) 291-307.

29 Smith, P.K., Krohn, R.I., Hermanson, G.T., Mallia, A.K., Gartner, F.H., Provenzano, M.D., Fujimoto, E.K., Goeke, M.N., Olson, B.J. and Klenk, D.C., Measurement of protein using bicinchoninic acid, Anal. Biochem., 150 (1985) 76-85.

30 Soria, B., Properties of miniature post-synaptic currents at the 
Torpedo marmorata nerve-electroplate junction, $Q$. J. Exp. Physiol., 68 (1983) 189-202.

31 Stadler, H. and Fenwick, E.M., Cholinergic synaptic vesicles from Torpedo marmorata contain an atractyloside binding protein related to the mitochondrial ADP/ATP carrier, Eur. J. Biochem., 136 (1983) 377-382.

32 Stadler, H. and Kiene, M.-L., Synaptic vesicles in electromotoneurones, II. Heterogeneity of populations is expressed in uptake properties; exocytosis and insertion of a core proteoglycan into the extracellular matrix, EMBO J., 6 (1987) 2217-2221.

33 Stanley, P.E. and Williams, S.G., Use of liquid scintillation spectrometer for determining adenosine triphosphate by the luciferase enzyme, Anal. Biochem., 29 (1969) 381-392.

34 Tashiro, T. and Stadler, H., Chemical composition of cholinergic synaptic vesicles from Torpedo marmorata based on improved purification, Eur. J. Biochem., 90 (1978) 479-487.

35 Weiler, M., Roed, I.S. and Whittaker, V.P., The kinetics of acetylcholine turnover in a resting cholinergic nerve terminal and the magnitude of the cytoplasmic compartment, J. Neurochem., 38 (1982) 1187-1191.

36 Whittaker, V.P., The vesicular basis of quantized transmitter release: a critical evaluation. In J.J. Feigenbaum and M. Hanani (Eds.), Presynaptic Regulation of Neurotransmitter Release: a Handbook, Freund, New York, 1989, in press.

37 Whittaker, V.P. and Barker, L.A., The subcellular fractionation of brain tissue with special reference to the preparation of synaptosomes and their component organelles. In R. Fried (Ed.), Methods of Neurochemistry, Vol. 2, Marcel Dekker, New York, 1972, pp. 1-52.
38 Whittaker, V.P., Essman, W.B. and Dowe, G.H.C., The isolation of pure cholinergic synaptic vesicles from the electric organs of elasmobranch fish of the family Torpedinidae, Biochem. J., 128 (1972) 833-846.

39 Wiedenmann, B. and Franke, W.W., Identification and localization of an integral membrane glycoprotein of $M_{r} 38,000$ (synaptophysin) characteristic of presynaptic vesicles, Cell, 41 (1985) $1017-1028$.

40 Zimmermann, H. and Denston, C.R., Recycling of synaptic vesicles in the cholinergic synapses of the Torpedo electric organ during induced transmitter release, Neuroscience, 2 (1977) 695-714.

41 Zimmermann, H. and Denston, C.R., Separation of synaptic vesicles of different functional states from the cholinergic synapses of the Torpedo electric organ, Neuroscience, 2 (1977) 715-730.

42 Zimmermann, H. and Whittaker, V.P., Effect of electrical stimulation on the yield and composition of synaptic vesicles from the cholinergic synapses of the electric organ of Torpedo: a combined biochemical, electrophysiological and morphological study, J. Neurochem., 22 (1974) 435-450.

43 Zimmermann, H. and Whittaker, V.P., Different recovery rates of the electrophysiological, biochemical and morphological parameters in the cholinergic synapses of the Torpedo electric organ after stimulation, J. Neurochem., 22 (1974) 1109-1114.

44 Zimmermann, H. and Whittaker, V.P., Morphological and biochemical heterogeneity of cholinergic synaptic vesicles, $\mathrm{Na}$ ture (Lond.), 267 (1977) 633-635. 